\title{
TYPE II MYOCARDIAL INFARCTION
}

\author{
Tripti Deb
}

In 2007, a joint Task Force of the American College of Cardiology, American Heart Association, European Society of Cardiology and the World Heart Federation published a redefinition of myocardial infarction (MI). Type-II MI was defined as MI secondary to ischemia due to either increased oxygen demand or decreased supply caused by conditions as coronary artery spasm, coronary embolism, anemia, arrhythmias, hypertension, or hypotension [1].In critically ill patients, or in patients undergoing major (non-cardiac) surgery, elevated values of cardiac biomarkers may appear, due to the direct toxic effects of endogenous or exogenous high circulating catecholamine levels. Also coronary vasospasm and/or endothelial dysfunction have the potential to cause MI. Most common causes with respect to frequency are anemia, sepsis, arrhythmia and postoperative status [2].

Numerous pathologies can cause disequilibrium in the balance between oxygen supply and demand. This balance is a critical determinant of the normal cardiac function, and understanding the variables involved is fundamental to understand the ischemic response in Type2 MI. Myocardial oxygen demand relies on three major determinants: systolic wall tension, contractility, and heart rate, whereas myocardial oxygen supply relies on the coronary blood flow and oxygen-carrying capacity. Disequilibrium in the myocardial supply and demand caused by alteration of factors involved in these complex hemodynamic interactions may lead to myocardial ischemia. For example, both hypoxia and anemia can decrease oxygen carrying capacity, leading to an imbalance secondary to supply mismatch.

This may result in ischemia, which, if severe enough, may lead to myocardial cell death with symptoms and/ or ECG changes and release of cTn [3]. If cTn exceeds the 99th percentile and there is a rising and/ or falling

Received on 15 OCT 2016, Published on 31 OCT 2016, Tripti Deb ${ }^{1}$

${ }^{1}$ Senior Consultant in Cardiology,

Apollo Hospitals, Hyderabad.

Corresponding Author: Tripti Deb

Email:_triptideb@yahoo.co.in pattern in conjunction with any other MI criteria in the absence of plaque rupture, this would then be, by definition, a T2MI.

In a study done by Stein et al, to assess the epidemiology, causes, management and outcomes of

type II MI patients, concluded that Type-II MI is not infrequent, especially among patients with NSTEMI. Compared to type-I MI, it is more frequent among the elderly with multiple comorbidities, high ACS risk score and associated with increased short and intermediateterm mortality. The implementation of current ACS guidelines to patients with type-II MI is challenging and more evidence-based patient-tailored therapeutic strategies are warranted [4].

The major limitations of this category include relatively small number of studies. Precise distinction between type II and type I MI in daily practice may be perplexing as many of the causes of type II MI may actually be a complication of type I MI (i.e. arrhythmia, heart failure) and other, such as surgery or inflammatory state may, by themselves, lead to plaque rupture.

The present study on type II MI patients concluded that patients with type-II MI have distinct demographics, associated with multiple comorbidities, a high-risk cardiovascular profile and this medical condition imposes a great therapeutic challenge and specific guidelines are necessary to manage this complex issue.

\section{REFERENCES:}

1. Thygesen K, Alpert JS, White HD (2007) Universal definition of myocardial infarction. Eur Heart J 28: 25252538.

2.Bertrand ME, LaBlanche JM, Tilmant PY, et al. Frequency of provoked coronary arterial spasm in 1089 consecutive patients undergoing coronary arteriography. Circulation. 1982; 65:1299-1306. 
3. Braunwald E.50th anniversary historical article. Myocardial oxygen consumption: the quest for its determinants and some clinical fallout. J Am Coll Cardiol. 1999 Nov 1;34(5):1365-8.
4. GY Stein, G Herscovici, R Korenfeld, et al. Type-II Myocardial Infarction - Patient Characteristics, Management and Outcomes. PLoSOne. 2014; 9(1): e84285. 